\title{
Distribuição e território das artérias cerebelares no encéfalo do gambá (Didelphis albiventris)
}

\author{
Distribution and territory of the cerebellar arteries on the opossum brain \\ (Didelphis albiventris)
}

Tânia Lindemann ${ }^{1}$ Rui Campos ${ }^{2}$

RESUMO

Utilizaram-se 30 encéfalos de Didelphis albiventris, com o sistema arterial preenchido com Látex 603 pigmentado. Sistematizaram-se as artérias cerebelares rostral, média e caudal, determinando a área territorial de irrigação. A artéria cerebelar rostral originou-se como continuação natural do ramo terminal da artéria basilar em 93,3\% dos casos, bilateralmente. percorrendo as faces rostral e dorsal dos hemisférios cerebelares, formando por sobre estes uma alça. A artéria cerebelar média, à direita, mostrou-se dupla em $70 \%$ e ímpar em $30 \%$ das peças; à esquerda, dupla em $56,7 \%$ e ímpar em $43,3 \%$ dos achados. A artéria cerebelar caudal apresentou-se, à direita, ímpar em 93,3\% e dupla em 6,7\% das peças; à esquerda, ímpar em $86,7 \%$ e dupla em $13,3 \%$ das amostras.

Palavras-chave: irrigação cerebelo, artérias cerebelares, gambá, marsupiais.

\section{ABSTRACT}

The rostral, middle and caudal cerebellar arteries were studied on the surface of the brain in 30 Didelphis albiventris, with the arterial system filled with colored latex. The arteries were systematized and the territorial area irrigation was determined. The rostral cerebellar artery was originated as a natural continuation of the terminal branch of the basilar artery in $93.3 \%$ of the cases, bilaterally, running to the rostral and dorsal surfaces of the lateral cerebellar hemispheres and forming a loop right above it. The middle cerebellar artery on the right side was seen double in $70 \%$ and single in $30 \%$ of the preparations and on the left side double in $56.7 \%$ and single in $43.3 \%$ of the findings. The caudal cerebellar artery was present single on the right side in $93.3 \%$ and double in $6.7 \%$ of the pieces and on the left side single in $86.7 \%$ and double in $13.3 \%$ of the cases.

Key words: cerebellar irrigation, cerebellar arteries, opossum, marsupials.

\section{INTRODUÇÃO}

Membros da família Didelphidea, comumente chamados gambás, apresentam-se amplamente distribuídos, com espécies que habitam o sul do Canadá, Estados Unidos, México, América Central e quase toda América do Sul. O gambá é praticamente onívoro, devorando pequenos roedores, aves e seus ovos, anfíbios, frutas e vegetais.

Do ponto de vista da anatomia desta espécie, ainda existem inúmeros aspectos que merecem estudos mais aprofundados. A irrigação encefálica constitui-se assunto de grande importância, uma vez que seu estudo encontra-se intimamente associado ao desenvolvimento do próprio sistema nervoso central. Em relação a este assunto, os dados da literatura clássica são inexistentes, e mesmo em trabalhos específicos os achados são escassos, sendo alguns deles imprecisos, principalmente devido ao número insuficiente de exemplares utilizados.

Tornou-se relevante portanto a comparação de nossos resultados com os de VORIS (1928), GILLILAN (1972) e LINDEMANN et al. (2000), que realizaram seus estudos em Didelphis $s p$. Na tentativa de compreender o desenvolvimento filogenético destes vasos, elaborou-se na discussão uma relação comparativa entre o grau de desenvolvimento estrutural do encéfalo na tartaruga, no gambá e no cão e as alterações territoriais vasculares que a partir daí se estabeleceram. A escolha da tartaruga e do cão para

\footnotetext{
${ }^{1}$ Médico Veterinário, Doutor, Professor Assistente de Anatomia Veterinária, Instituto de Ciências Básicas da Saúde (ICBS), Departamento de Ciências Morfológicas (DCM), Universidade Federal do Rio Grande do Sul (UFRGS). Rua Gal. Couto de Magalhães 1155, apto.1002, 90540-131, Porto Alegre, RS. E-mail: lindeman@orion.ufrgs.br. Autor para correspondência.

${ }^{2}$ Médico Veterinário, Doutor, Professor Adjunto de Anatomia Veterinária, ICBS, DCM, UFRGS.
} 
comparação deveu-se à distancia relativa entre eles e o gambá, em termos de evolução na escala zoológica e pela característica comum de serem animais macrosmáticos.

Para tanto, estabeleceu-se dados comparativos com os trabalhos de BURDA (1965), GILLILAN (1967) e FRIZZO et. al. (1994), em tartarugas, e os relatos de DELLMANN \& MC CLURE (1981), NANDA (1981), BEITZ \& FLETCHER (1993) e EVANS (1993), em Canis familiaris.

Objetivamos neste estudo descrever o comportamento, a distribuição e o território das artérias cerebelares na superfície do encéfalo em Didelphis albiventris (gambá). Além disso, explicar como a evolução da irrigação encefálica acompanhou o desenvolvimento do cerebelo, principalmente no que se refere às novas aquisições evolutivas.

\section{MATERIAL E MÉTODO}

Foram utilizados 30 encéfalos com segmento de medula espinhal de Didelphis albiventris, 14 machos e 16 fêmeas, capturados em vários municípios do RS, sob licença $n^{\circ}$ 079/98 e 270/99, processo $n^{\circ}$ 1887/98-99, do Instituto Brasileiro do Meio Ambiente e Recursos Renováveis - IBAMA. Os animais foram previamente tranqüilizados com éter sulfúrico $^{\mathrm{a}}$, pesados e anestesiados com tiopental sódico $^{\mathrm{b}}$, por via intraperitonial, na dose média de $150 \mathrm{mg} / \mathrm{kg}$, e imediatamente após cada animal recebeu 2500 UI de heparina ${ }^{c}$, pela mesma via. A seguir foi feita a abertura da parede torácica, para exposição do coração que foi então seccionado, permitindo a canulação do tronco braquiocefálico através do ventrículo esquerdo e artéria aorta. Imediatamente após foi procedida a lavagem do sistema arterial, com solução salina resfriada, seguindo-se o preenchimento deste com uma mistura de Látex $603^{\mathrm{d}}$, corado em vermelho com pigmento específico ${ }^{\mathrm{e}}$.

As peças permaneceram aproximadamente uma hora sob água corrente, para o resfriamento e solidificação do material injetado, após foi retirada a pele da cabeça e aberta uma janela óssea na abóbada craniana. Em seguida, as peças foram imersas em formaldeído $20 \%$, durante um período mínimo de 170 horas.

Após a retirada do encéfalo com segmento de medula espinhal cervical, as peças foram dissecadas para permitir a observação e o registro do comportamento dos vasos. Com vistas à documentação, foram feitos esquemas de todas as preparações e fotos de algumas delas.

\section{RESULTADOS}

A artéria cerebelar rostral era a mais calibrosa dentre as artéria cerebelares. Apresentou-se como a continuação natural do ramo terminal da artéria basilar, após sua anastomose com a artéria comunicante caudal ipsilateral, ramo caudal da artéria carótida interna. Contornou lateralmente o tronco encefálico, surgindo na face dorsal deste, laterocaudalmente ao colículo caudal (Figuras 1 e 2). Logo que alcançou a face dorsal do tronco encefálico lançou como colateral a artéria tectal caudal e continuou-se naturalmente para o cerebelo, contornado dorsalmente a base do flóculo, descendo aos lóbulos caudais do vermis para o interior do quarto ventrículo, neste ponto seus ramos terminais anastomosaram-se com sua homóloga contralateral. Em seu percurso, formou uma alça por sobre a superfície do cerebelo e emitiu inúmeros ramos colaterais que se distribuíram geralmente pelo interior das fissuras cerebelares para o vermis, hemisférios cerebelares e para o plexo corióide do quarto ventrículo.

O comportamento padrão ocorreu em 93,3\% das peças, bilateralmente. Em 6,7\% dos casos, em ambos os antímeros, a artéria cerebelar rostral após atingir a face rostral dos hemisférios cerebelares, não apresentou sua forma característica de alça, ramificando-se logo em seguida e seus finos ramos mergulharam diretamente nas fissuras do cerebelo. Nestes encéfalos, ela não era, portanto, visível na superfície dorsolateral do hemisfério cerebelar.

A artéria tectal caudal era o ramo colateral da artéria cerebelar rostral. Emergiu desta no nível da porção caudal do tecto mesencefálico, avançou medialmente em direção ao colículo caudal, para se distribuir no terço caudal deste (Figura 1). No antímero direito, esta artéria mostrou-se ímpar em 83,3\% das peças, enquanto em $16,7 \%$ das preparações apresentou-se duplicada. No antímero esquerdo, apresentou-se ímpar em $86,7 \%$ dos casos, duplicada em $10 \%$ e ausente em $3,3 \%$, neste caso a artéria tectal rostral cobriu o território de distribuição da artéria tectal caudal.

O território da artéria cerebelar rostral compreendeu o terço caudal do colículo caudal, os lóbulos rostral, médio e caudal do vermis, bem como as faces rostral e caudal dos hemisférios cerebelares. Contribuiu ainda com ramos discretos para o plexo corióide do quarto ventrículo.

A artéria cerebelar média apresentou-se geralmente duplicada, constituída de um vaso mais rostral e outro mais caudal. $\mathrm{O}$ vaso mais rostral era inconstante e originava-se do ramo terminal ipsilateral 
da artéria basilar, dirigindo-se para a face rostral do cerebelo, irrigando os lóbulos rostroventrais (Figura 2). O vaso mais caudal era constante e mais calibroso, originado da artéria basilar ao nível da ponte (Figura 2). Projetava-se lateral e dorsalmente até alcançar a parte ventral do flóculo, no nível da origem aparente dos nervos cranianos VIIo e VIII ${ }^{\circ}$, anastomosou-se com ramos da artéria cerebelar caudal e distribuiu-se no flóculo, também contribuindo discretamente para a irrigação do plexo corióide do quarto ventrículo. No antímero direito, a apresentação padrão ocorreu em $70 \%$ das amostras e em $30 \%$ dos casos apresentou apenas o vaso mais caudal. No antímero esquerdo, esta artéria mostrou-se duplicada em 56,7\% das preparações e em 43,3\% das peças surgiu como uma artéria ímpar, persistindo o vaso mais caudal.

O território da artéria cerebelar média, quando esta se apresentou duplicada, na maioria dos casos, compreendeu a porção rostral e lateral do hemisfério cerebelar, a parte ventral do flóculo, a região da origem aparente dos nervos cranianos VIIo e VIII - e contribuiu discretamente para o plexo corióide do quarto ventrículo. Quando esta artéria apresentou apenas o vaso mais caudal, o território que seria irrigado pelo vaso mais rostral foi assumido pela artéria cerebelar rostral, que logo após sua origem lançou, nestes casos, um ramo para esta porção.

A artéria cerebelar caudal originou-se como ramo colateral da artéria basilar, na altura de seu terço médio. Projetou-se lateralmente até alcançar a origem aparente dos pares cranianos VIIo e VIII , onde lançou um pequeno ramo para anastomose com o vaso mais caudal da artéria cerebelar média (Figura 1). A partir daí, seguiu em direção dorsal, contornou a medula oblonga indo distribuir-se em sua face dorsal, nos lóbulos caudais do vermis, constituindo a principal fonte para o plexo corióide do quarto ventrículo. Apresentou-se, à direita, ímpar em 93,3\% das peças e em 6,7\% das 30 preparações, mostrou-se dupla. Já à esquerda, esta artéria mostrou-se ímpar em $86,7 \%$ dos 30 encéfalos e em $13,3 \%$ das amostras apareceu duplicada.

O território da artéria cerebelar caudal compreendeu a porção ventrocaudal dos hemisférios cerebelares e os lóbulos mais caudais do vermis, e formou a principal fonte de suprimento sanguíneo para o plexo corióide do quarto ventrículo.

\section{DISCUSSÃO}

Conforme previamente referido, apenas na literatura especializada foram encontradas citações relativas à irrigação encefálica do Didelphis albiventris, e ainda mais escassos foram os dados concernentes à distribuição dos vasos encefálicos e seus territórios de irrigação.

Em relação à irrigação para o cerebelo, a artéria cerebelar rostral constituiu a principal fonte. Este vaso originou-se como a continuação natural do ramo terminal da artéria basilar, após receber anastomose com a artéria comunicante caudal (LINDEMANN et al., 2000). Ao atingir a face lateral do cerebelo, formou na superfície deste uma alça. Para VORIS (1928), a origem da artéria cerebelar rostral era semelhante aos nossos achados, porém o autor considerou a divisão deste vaso em um ramo rostral e um ramo caudal. O ramo rostral correspondia à artéria tectal caudal de nossos resultados, e o ramo caudal correspondia a continuação da própria artéria cerebelar rostral, que se encurvava caudalmente lançando ramos para as faces rostral e ventral dos lobos médio e laterais do cerebelo. Citou ainda a presença de uma anastomose com a artéria cerebelar média, a qual não foi visualizada em nossas preparações. GILLILAN (1972) também considerou a artéria cerebelar rostral como a principal fonte de irrigação para o cerebelo, este vaso originou-se como o mais caudal dos ramos que surgiu da bifurcação rostral da artéria basilar. Em seu trajeto, lançou ramos marginais para a face ventral do lobo lateral e para o pedúnculo cerebelar médio, um ramo tectal e ramos corticais cujas ramificações estenderam-se caudalmente suprindo os lobos laterais e médio do cerebelo. $\mathrm{O}$ primeiro ramo, que se dirigiu para a face ventral do lobo lateral, correspondia, em nossas preparações, ao ramo lançado pela cerebelar rostral quando a cerebelar média era ímpar, ou seja, na ausência do vaso mais rostral. Nenhum destes dois autores comentou sobre a formação de uma alça que percorria as faces rostral e caudal dos hemisférios cerebelares.

A artéria tectal caudal surgiu da artéria cerebelar rostral na lateral do colículo caudal, avançava em direção dorsal suprindo o terço caudal deste. VORIS (1928) denominou este vaso de ramo rostral da cerebelar rostral e afirmou que este ramificava-se nas faces dorsal e rostral dos corpos quadrigêmeos, suprindo-os e anastomosando-se com ramos da artéria cerebelar caudal. Para GILLILAN (1972), os ramos tectais originavam-se dos troncos basilares e passavam ao redor do cérebro médio no nível do colículo rostral, portanto mais rostralmente que em nossas peças. Estas artérias junto com os ramos tectais da artéria cerebral caudal e com as artérias cerebelares rostrais formavam um plexo sobre os corpos quadrigêmeos. Nas peças aqui pesquisadas, a distribuição desta artéria apresentava aspecto arboriforme e não plexiforme. 


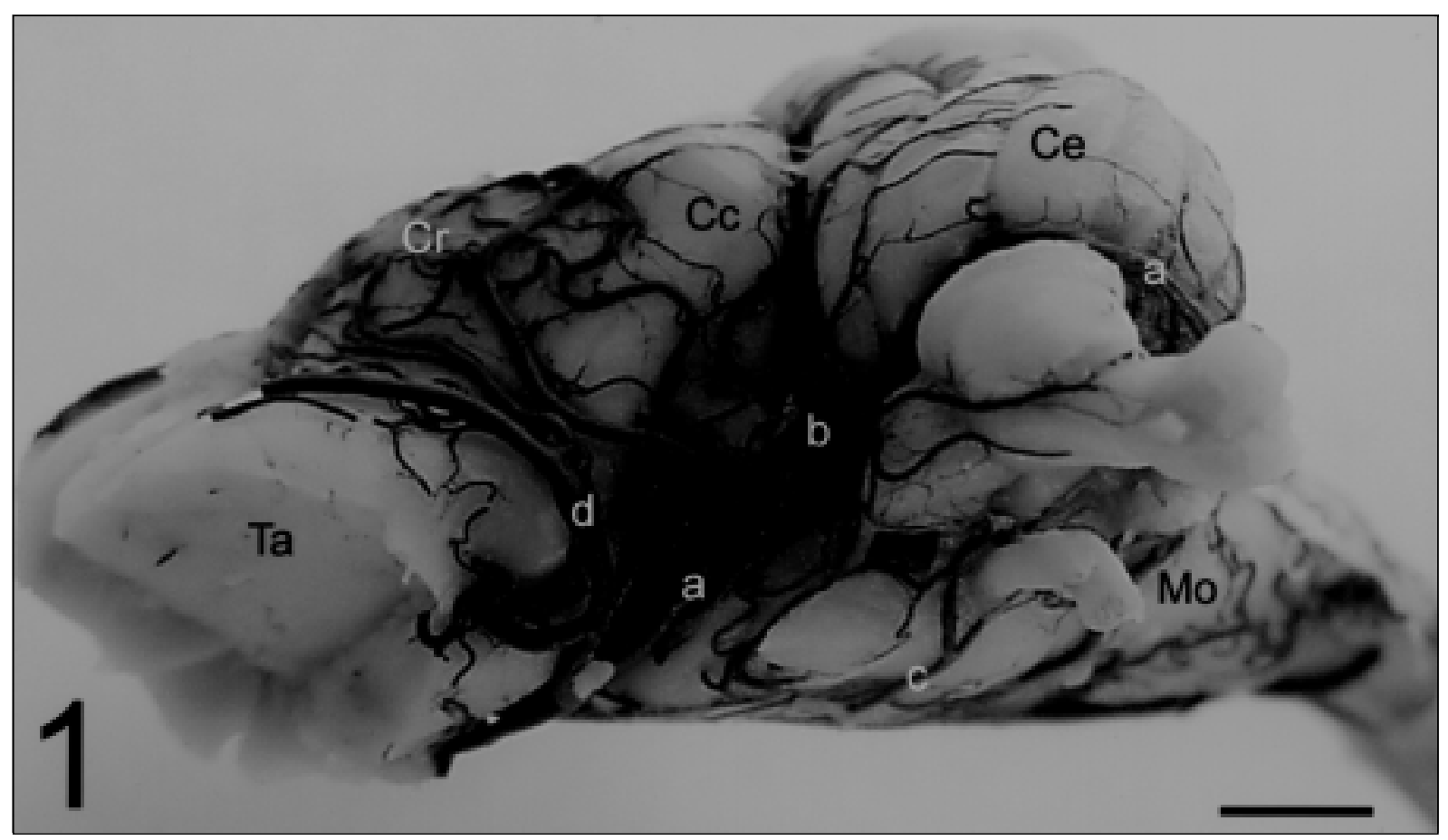

Figura 1 - Fotografia em detalhe do terço caudal do encéfalo de Didelphis albiventris, vista lateral, antímero esquerdo, mostrando distribuição das artérias cerebelares rostral e caudal. a- artéria cerebelar rostral; b- artéria tectal caudal; c- artéria cerebelar caudal; d- artéria cerebral caudal; e- artéria tectal rostral; Ta- tálamo; Cr- colículo rostral; Cc- colículo caudal; Ce- cerebelo; Momedula oblonga. Barra $=0,3 \mathrm{~cm}$.

O território da artéria cerebelar rostral compreendeu os lobos rostral, médio e caudal do vermis, as faces rostral e caudal dos hemisférios cerebelares (VORIS, 1928; GILLILAN, 1972). Também pertence a seu território o terço caudal do colículo caudal e o plexo corióide do quarto ventrículo.

A artéria cerebelar média apresentou-se geralmente duplicada, constituída de um vaso mais rostral e outro mais caudal. O vaso mais rostral, inconstante, originou-se do ramo terminal ipsilateral da basilar, enquanto o vaso mais caudal, constante e mais calibroso, surgiu diretamente da artéria basilar no nível da ponte (LINDEMANN et al., 2000). O vaso mais rostral dirigiu-se para a face rostral do cerebelo, irrigando os lóbulos rostroventrais. $\mathrm{O}$ vaso mais caudal projetou-se lateral e dorsalmente em direção ao flóculo, também contribuindo com pequenos ramos para o plexo corióide do quarto ventrículo. Para VORIS (1928), a artéria cerebelar média, por ele denominada de artéria cerebelar ântero-inferior, era o ramo mais rostral da artéria basilar, projetando-se lateralmente, suprindo a porção rostral do flóculo e as partes lateral e rostral dos lobos laterais do cerebelo e para o plexo corióide do quarto ventrículo. GILLILAN (1972) afirmou que, no gambá, apenas um par de artérias caudais supria o cerebelo, originando-se simetricamente da porção média da artéria basilar, porém referiu-se a um outro ramo que supria a face caudal dos lobos laterais e médio do cerebelo, anastomosando-se com ramos da artéria cerebelar rostral. Apesar de o autor não citar especificamente a artéria cerebelar média, parece-nos que esta descrição correspondia ao referido vaso.

A artéria cerebelar caudal originou-se como ramo colateral da artéria basilar, em seu terço médio (LINDEMANN et al., 2000). Projetou-se dorsal e lateralmente distribuindo-se nos lóbulos caudais do vermis, e constituindo a principal fonte de irrigação para o plexo corióide do quarto ventrículo. Para VORIS (1928), a artéria cerebelar caudal podia originar-se da artéria basilar junto à origem desta, emergir da artéria vertebral, pouco antes da união com sua homóloga para formar a basilar, ou ainda resultar da fusão de dois ramos que emergiram em cada um desses modos. Este vaso supria a parte dorsal da medula, as partes caudal e ventral do vermis cerebelar, incluindo o nódulo e as partes caudais dos lobos laterais. GILLILAN (1972) observou um par de artérias caudais para suprir o cerebelo, que surgiram simetricamente no meio da artéria basilar, estendendo- 


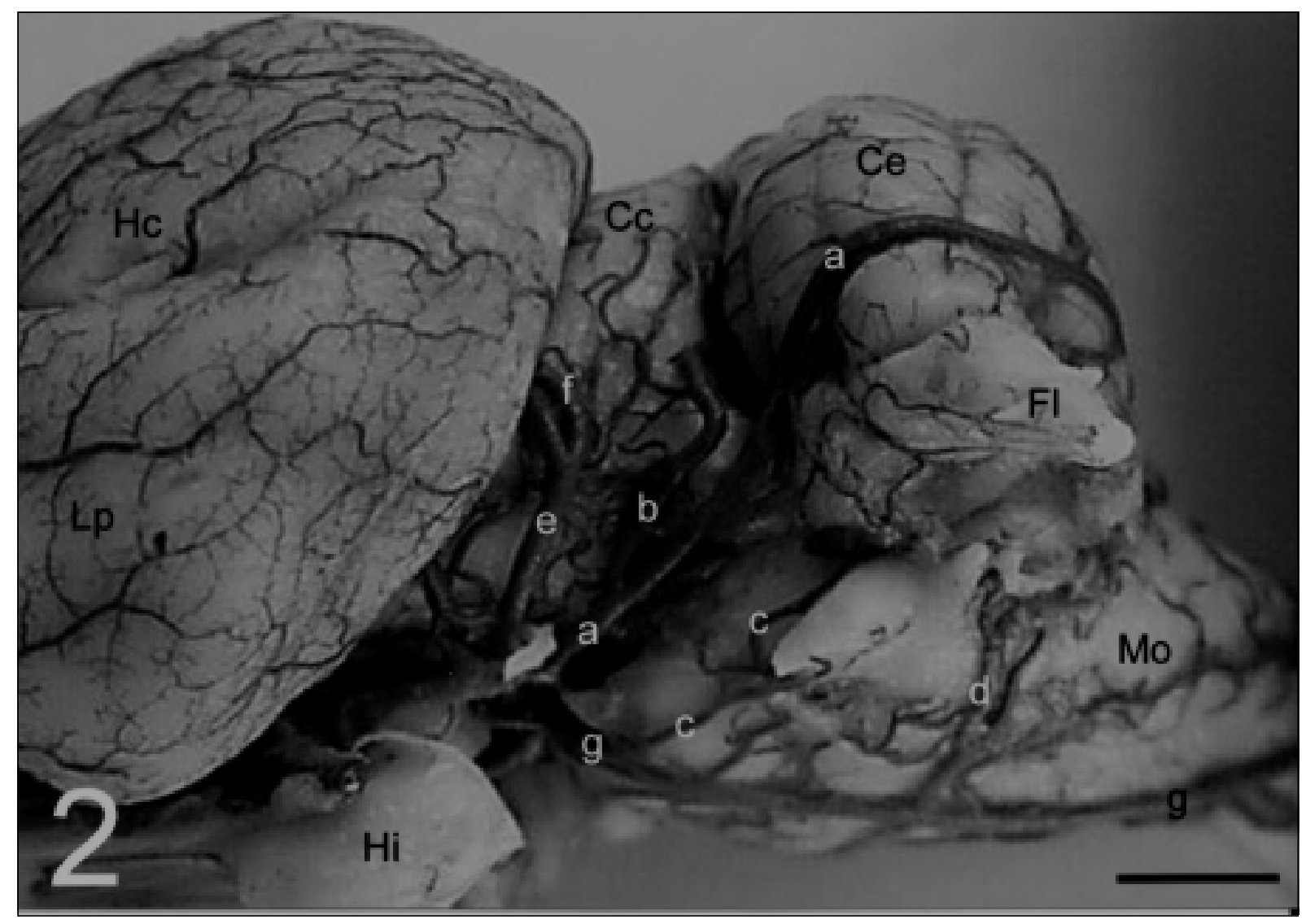

Figura 2 - Fotografia da vista lateroventral, antímero esquerdo, do encéfalo de Didelphis albiventris. a- artéria cerebelar rostral; b- artéria tectal caudal; c- artéria cerebelar média; d- artéria cerebelar caudal; e- artéria cerebral caudal; f- artéria tectal rostral; g- artéria basilar; Lp- lobo piriforme; Hc- hemisfério cerebral; Hi- hipófise; Cc- colículo caudal; Ce- cerebelo; Fl- flóculo; Mo- medula oblonga. Barra $=0,3 \mathrm{~cm}$.

se da borda caudal do cerebelo, lançando ramos para o plexo corióide do quarto ventrículo e para o flóculo. Comentou ainda que outro ramo supria a face caudal dos lobos laterais e médio do cerebelo.

A irrigação do cerebelo na filogênese envolveu dois tipos básicos de mudanças: um primeiro estrutural, de crescimento, com a aquisição dos hemisférios cerebelares, adquiridos apenas nos mamíferos (BECCARI, 1943); e um segundo movimento evolutivo, envolvendo a origem do fluxo sangüíneo, com a mudança da fonte básica proveniente do sistema carotídeo para fonte de origem vértebrobasilar (DE VRIESE, 1905 e LINDEMANN et al., 2000). Segundo BURDA (1965) e FRIZZO et al. (1994), na tartaruga, o encéfalo todo era suprido pela fonte carotídea e o cerebelo, que se resumia apenas a uma pequena saculação correspondente ao primórdio do vérmis médio, era irrigado pelas artérias mesencefálica e cerebelar. No gambá, o cerebelo apresentou hemisférios cerebelares, ainda pouco desenvolvidos e um vermis bem estruturado; três pares de artérias provenientes da artéria basilar ou de seus ramos terminais faziam a sua irrigação (LINDEMANN et al., 2000). A modificação aqui adquirida consistiu no avanço do sistema vértebro-basilar, que passou a fornecer sangue para o cerebelo e porção caudal do tronco encefálico. Segundo relatos de DELLMANN \& MC CLURE (1981) e BEITZ \& FLETCHER (1993), no cão o cerebelo apresentou um maior crescimento dos hemisférios cerebelares, porém persistiram três pares de artérias cerebelares, provenientes da artéria basilar ou de seus ramos terminais e o fluxo sangüíneo igualmente persistiu fornecido pelo sistema vértebro-basilar (NANDA, 1981 e EVANS, 1993).

\section{FONTES DE AQUISIÇÃO}

a -Technion Indústrias Químicas Exportadora Ltda
b -E. Merck A.G.

c -Liquemine Roche

Ciência Rural, v.34, n.2, mar-abr, 2004. 
d -Bertoncini

e -Suvinil corante - Basf S.A.

\section{REFERÊNCIAS BIBLIOGRÁFICAS}

BECCARI, N. Neurologia comparata - anatomo-funzionale dei vertebrati, compreso l'uomo. Firenze : Sansoni Edizioni Scientifiche, 1943. 777p.

BEITZ, A.J.; FLETCHER, T.F. The Brain. In: EVANS, H.E. Miller's anatomy of the dog. 3.ed. Philadelphia : Saunders, 1993. Cap.18, p.894-952.

BURDA, D. Development of intracranial arterial patterns in turtles. Journal of Morphology, New York, v.116, p.171-188, 1965.

DELlMANN, H.D.; McClURE, R.C. Sistema nervoso do carnívoro. In: GETTY, R. Anatomia dos animais domésticos. 5.ed. Rio de Janeiro : Interamericana, 1981. V.2, cap.57, p.15691595.

DE VRIESE, B. Sur la signification morphologique des artères cérébrales. Archives de Biologie, v. 21, p.357-457, 1905.

EVANS, H.E. The hart and the arteries. In: Miller's anatomy of the dog. 3.ed. Philadelphia : Saunders, 1993. Cap.11, p.586-681.
FRIZZO, M.E.S. et al. The vasculature of the subfornical organ of the turtle Chrysemys dorbigni. Italian Journal of Anatomy and Embriology, Firenze, v.99, p.109-121, 1994.

GILLILAN, L. A comparative study of the extrinsic and intrinsic arterial blood supply to brains of the submammalian vertebrates. Journal of Comparative Neurology, Philadelphia, v.130, p.175196, 1967.

GILLILAN, L. Blood supply to primitive mammalian brains. Journal of Comparative Neurology, Philadelphia, v.145, p.209222, 1972.

LINDEMANN, T.; RECKZIEGEL, S.; CAMPOS, R. A systematic study of brain base arteries in the opossum Didelphis albiventris. Brazilian Journal of Morphological Sciences, São Paulo, v.17, p.35-41, 2000.

NANDA, B.S. Suprimento sangüíneo ao encéfalo. In: GETTY, R. Anatomia dos animais domésticos. 5.ed. Rio de Janeiro : Interamericana, 1981. V.2, cap.55, p.1513-1518.

NOMINA ANATOMICA VETERINARIA. 4.ed. New York : International commitee on veterinary gross anatomical nomenclature, 1994. $198 \mathrm{p}$.

VORIS, H.C. The arterial supply of the brain and spinal cord of the virginian opossum (Didelphis virginiana). Journal of Comparative Neurology, Philadelphia, v.44, p.403-423, 1928. 